\title{
The Analysis of Closed-form Solution for Energy Detector Dynamic Threshold Adaptation in Cognitive Radio
}

\author{
Rade BOZOVIC ${ }^{1}$, Mirjana SIMIC ${ }^{1}$, Predrag PEJOVIC ${ }^{2}$, Miroslav L. DUKIC ${ }^{3}$ \\ ${ }^{1}$ Dept. of Telecommunications, Faculty of Electrical Engineering, University of Belgrade, Bulevar Kralja Aleksandra 73, \\ 11120 Belgrade, Serbia \\ ${ }^{2}$ Dept. of Electronics, Faculty of Electrical Engineering, University of Belgrade, Bulevar Kralja Aleksandra 73, \\ 11120 Belgrade, Serbia \\ ${ }^{3}$ Dept. of Telecommunications, Singidunum University, Danijelova 32, 11120 Belgrade, Serbia \\ radepremijer@gmail.com, \{mira,peja\}@etf.rs, mdukic@singidunum.ac.rs
}

Submitted February 6, 2017 / Accepted April 7, 2017

\begin{abstract}
Spectrum sensing is the most important process in cognitive radio in order to ensure interference avoidance to primary users. For optimal performance of cognitive radio, it is substantial to monitor and promptly react to dynamic changes in its operating environment. In this paper, energy detector based spectrum sensing is considered. Under the assumption that detected signal can be modelled according to an autoregressive model, noise variance is estimated from that noisy signal, as well as primary user signal power. A closed-form solution for optimal decision threshold in dynamic electromagnetic environment is proposed and analyzed.
\end{abstract}

\section{Keywords}

Cognitive radio, energy detector, noise variance estimation, spectrum sensing, threshold adaptation

\section{Introduction}

Based on one of popular definitions [1], cognitive radio (CR) is an intelligent wireless communication system that is aware of its ambient environment. This CR will learn from the environment and adapt its internal states to statistical variations in the existing RF stimuli by adjusting the transmission parameters (frequency band, modulation mode and transmit power) in real-time. Sensing function and ability to promptly modify its transmission parameters are unique features and challenges of cognitive radio implementation [2], [3].

Various methods have been proposed for spectrum sensing in CR, which include matched filter, cyclostationary detection, and energy detection [4]. Matched filter and cyclostationary detection are coherent detection techniques, with better detection performance than non-coherent energy detection. However, coherent detectors require a priori knowledge about the signal of the primary users (PU) [5].
Matched filter [6] is the optimal detection technique since it maximizes the signal to noise ratio (SNR), but requires demodulation parameters of the PU signal. Cyclostationary detector [7] can detect very weak signals on the basis of their cyclic features, but information about the cyclic characteristics is needed. Non-coherent energy detector [8] is based on comparison of the detected signal energy with the decision threshold. This method does not require any knowledge about the PU signal, and has low implementation complexity compared to matched filter and cyclostationary detection. In addition, the energy detection can provide lower sensing time than the other methods (in the high SNR environments) and can be applied for wideband sensing. On the other hand, the energy detector has many disadvantages such as high sensitivity of the threshold level to variations of the noise level, high sensing time in the low SNR environments, it cannot distinguish primary and secondary user signals as well as signals and noise, and cannot detect spread spectrum signals. Despite the numerous drawbacks, energy detection is the most popular and the most studied technique among all spectrum sensing methods.

The most important process that affects performance of energy detectors is the threshold selection. Various double threshold methods [9], [10] are proposed to improve the threshold selection process. Cooperative sensing with multiple CRs is suggested in [11], while in [12] spectrum utilization factor is applied to minimize the total error sensing probability. One solution to improve the performance of the energy detector is to make use of time reversal technique, what was analyzed in ultra-wideband systems [13]. Application of the energy detector method for spectrum sensing with the use of random sampling is evaluated in a comparison with the uniform sampling case in [14]. Exact closed-form expression for the optimal detection threshold for different receiver diversity systems was derived in [15]. Improved reliability with multiple threshold values determination by using fuzzy entropy maximization was suggested in [16]. In [17], authors propose an algorithm for 


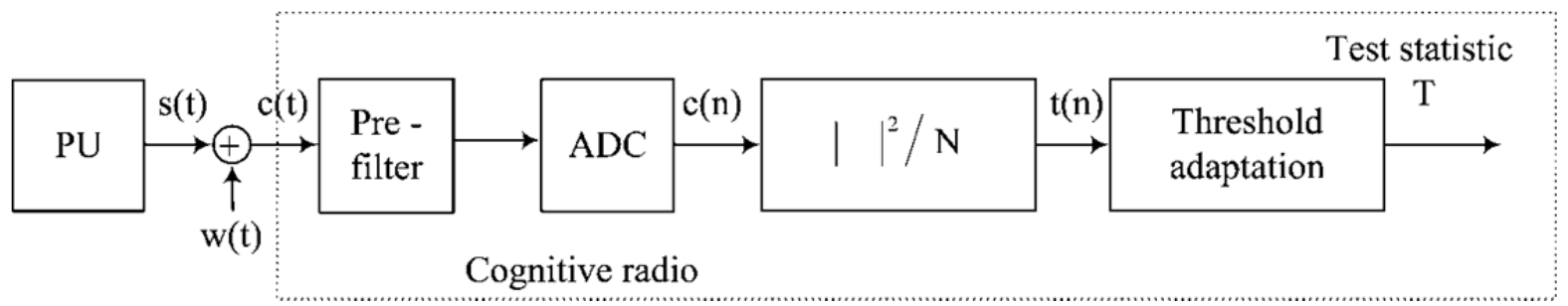

Fig. 1. System model of energy detector based CR.

energy detection threshold adaptation assuming that the noise power is completely known at the cognitive user. Also, many algorithms use a fixed decision threshold to distinguish PU signals from the noise. For example, an experimental threshold is set in [8] by measuring the noise power. However, in practical systems, the noise power fluctuates due to the temperature changes, ambient interference, and it is difficult to guarantee the detection probability and the false alarm probability with the fixed threshold setting.

In order to achieve better performance and to improve reliability of detection, $\mathrm{CR}$ as environment-aware system should dynamically adapt the decision threshold according to the changes in the environment. In [18], energy-based spectrum sensing in dynamic scenarios is studied and an iterative gradient-based algorithm for sensing the threshold adaptation is proposed. The iterative approach is often impractical, since it requires an initial estimate to ensure global convergence. Also, iterative approach can be computationally inefficient. On the other hand, closed-form solution provides optimal decision threshold in a single step and does not require initial estimate values, resulting in a lower spectrum sensing time. Also, closed-form solution is more efficient from the viewpoint of arithmetical operations, which improves general performance of the cognitive radio spectrum sensing process. This motivated the work presented in this paper, which studies energybased spectrum sensing in dynamic scenarios, and proposes and analyses a closed-form algorithm for the sensing threshold adaptation, assuming that the noise power is not known at the cognitive user.

The paper is organized as follows: System model and general problem description are presented in Sec. 2. Noise and PU signal variance estimation are given in Sec. 3. Threshold determination and analysis of closed-form solution are presented in Sec. 4. Simulation results are shown in Sec. 5. The main conclusion of the work is given in Sec. 6 .

\section{System Model}

Environment with the PU as an M-quadrature amplitude modulation (16-QAM) signal is considered. Transmitted signals are passed through the channel with additive white Gaussian noise (AWGN). From the detected input signal ( $N$ number of samples), CR estimates variances of the noise and the PU signal. According to the estimated parameters, CR determines the threshold level and performs test statistic for spectrum sensing. The system model is schematically described in the block diagram of Fig. 1.
After the $\mathrm{CR}$ processing along with the analog to digital conversion (ADC), the received signal $c(n)$ can be expressed as

$$
c(n)=s(n)+\omega(n),
$$

with $s(n)$ being the active radio signal generated by the PU and $\omega(n)$ the AWGN, with zero mean and variance $\sigma_{\omega}{ }^{2}(n)$. Signal $s(n)$ can be considered as a Gaussian random process, with variance $\sigma_{\mathrm{s}}^{2}(n)$ [19].

Test statistic for the energy detector is implemented as the time average of the received signal energy

$$
t(n)=\frac{1}{N} \sum_{i=n-N}^{n}|c(i)|^{2},
$$

with $t(n)$ as an average power of the received CR signal at the time instant $n$. Binary hypothesis is performed at any given time instant $n$ in order to estimate occupancy of the frequency band, where $H_{0}$ and $H_{1}$ denote hypothesis of the absence and the presence of the PU signal, respectively, as formulated by

$$
\begin{gathered}
H_{0}: c(n)=\omega(n), \\
H_{1}: c(n)=s(n)+\omega(n) .
\end{gathered}
$$

The decision rule is given by

$$
t(n) \underset{<}{H_{1}} \underset{H_{0}}{<} \gamma(n)
$$

where $\gamma(n)$ is the decision threshold determined at the time instant $n$. Performance of the spectrum sensing can be evaluated through the probability of detection of active PU, $P_{\mathrm{d}}$, and the probability of false alarm for presence of active $\mathrm{PU}, P_{\mathrm{fa}}$, which is given in the terms of $Q$ function [19] as

$$
\begin{gathered}
P_{\mathrm{d}}(\gamma(n))=P_{\mathrm{r}}\left(t(n)>\gamma(n) \mid H_{1}\right)= \\
Q\left(\sqrt{\left.\frac{N}{2} \frac{\gamma(n)-\left(\sigma_{\omega}^{2}(n)+\sigma_{\mathrm{s}}^{2}(n)\right)}{\sigma_{\omega}^{2}(n)+\sigma_{\mathrm{s}}^{2}(n)}\right),}\right. \\
P_{\mathrm{fa}}(\gamma(n))=P_{\mathrm{r}}\left(t(n)<\gamma(n) \mid H_{0}\right)= \\
Q\left(\sqrt{\left.\frac{N}{2} \frac{\gamma(n)-\sigma_{\omega}^{2}(n)}{\sigma_{\omega}^{2}(n)}\right) .}\right.
\end{gathered}
$$


Based on (6) and (7), performance of the spectrum sensing at any time instant $n$ highly depends on the threshold value $\gamma(n)$. Higher $P_{\mathrm{d}}$ means less harmful interference to PU and less $P_{\mathrm{fa}}$ means less missed transmission opportunities and therefore better spectrum utilization. Large value of $P_{\mathrm{d}}$ and small value of $P_{\mathrm{fa}}$ are the design goals. Improving one of these performance indicators implies degradation of the other one, so a trade-off between these two parameters is determined by $\gamma(n)$. To optimize the spectrum sensing process, both $P_{\mathrm{d}}$ and $P_{\mathrm{fa}}$ should be considered, and they are combined into a single criterion - spectrum sensing error (SSE), $\varepsilon(\gamma(n))[20]$ as

$$
\varepsilon(\gamma(n))=\alpha P_{\mathrm{fa}}(\gamma(n))+(1-\alpha)\left[1-P_{\mathrm{d}}(\gamma(n))\right]
$$

where $\alpha(0<\alpha<1)$ is a weighting factor for the probability of the false alarm relative to the probability of detection.

The goal is to minimize SSE in order to improve performance of the energy based spectrum sensing in dynamic scenarios. In this paper, we propose a closed-form solution for the optimal decision threshold $\gamma(n)$ and a dynamic adaptation to time variation of the PU signal and unknown noise, causing the system to respond to the changes in the environment.

\section{Estimation of Noise and PU Power}

Under the assumption that the signal detected by CR can be modelled according to an autoregressive (AR) model of the order $p$, then the noise power level, which is equal to the noise variance $\sigma_{\omega}{ }^{2}(n)$, can be estimated from the noisy AR signal [21]. PU signal $s(n)$ and the noise signal $\omega(n)$ are uncorrelated, thus PU signal variance $\sigma_{\mathrm{s}}{ }^{2}(n)$ can be estimated based on the calculated variance of CR signal $\sigma_{\mathrm{c}}^{2}(n)$ as

$$
\sigma_{\mathrm{S}}^{2}(n)=\sigma_{\mathrm{c}}^{2}(n)-\sigma_{\omega}^{2}(n) .
$$

Transfer function of the $p$-th order AR model representation of the signal $s(n)$ is

$$
H(z)=\frac{1}{1+\sum_{j=1}^{p} a_{j} z^{-j}}
$$

where $a_{j}$ are parameters of the AR model, estimated by using the least squares procedure, and they satisfy the set of the Yule-Walker equations [22]. Relation between the autocorrelation coefficients of the uncontaminated signal $s(n)$ and the $\mathrm{CR}$ detected signal $c(n)$ is given as [22]

$$
\begin{aligned}
& R_{\mathrm{S}}(0)=R_{\mathrm{c}}(0)-\sigma_{\omega}^{2}, \\
& R_{\mathrm{S}}(j)=R_{\mathrm{c}}(j), j>0
\end{aligned}
$$

where $R_{\mathrm{s}}(j)$ and $R_{\mathrm{c}}(j)$ represent autocorrelation coefficients of the uncontaminated signal $s(n)$ and CR signal $c(n)$, respectively.
Finally, from (11) and (12) the noise power estimation is given by

$$
\sigma_{\omega}^{2}=\frac{\sum_{j=1}^{p} a_{j}\left(\hat{R}_{\mathrm{c}}(j)+\sum_{i=1}^{p} a_{i} \hat{R}_{\mathrm{c}}(|j-i|)\right)}{\sum_{j=1}^{p} a_{j}^{2}}
$$

where $\hat{R}_{\mathrm{c}}(j)$ is an estimate of the autocorrelation coefficients of the CR signal $c(n)$, obtained from an overdetermined set of $q>p$ high order Yule-Walker equations using the least squares procedure [22].

\section{Threshold Determination and Dynamic Adaptation}

Function of the SSE, specified by (8), is strictly convex function of the threshold $\gamma(n)$, for given value of $\alpha$. Next, let $\partial \varepsilon(\gamma(n)) / \partial \gamma(n)=0$ and according to (6), (7) and (8) a quadratic equation for the decision threshold is obtained

$$
\begin{aligned}
& \frac{2 \sigma_{\omega}^{2}(n)+\sigma_{\mathrm{S}}^{2}(n)}{\sigma_{\omega}^{2}(n)\left(\sigma_{\omega}^{2}(n)+\sigma_{\mathrm{S}}^{2}(n)\right)} \gamma^{2}(n)-2 \gamma(n)- \\
& \frac{4 \sigma_{\omega}^{2}(n)\left(\sigma_{\omega}^{2}(n)+\sigma_{\mathrm{S}}^{2}(n)\right)}{N \sigma_{\mathrm{S}}^{2}(n)} \ln \left(\frac{\alpha\left(\sigma_{\omega}^{2}(n)+\sigma_{\mathrm{s}}^{2}(n)\right)}{(1-\alpha) \sigma_{\mathrm{S}}^{2}(n)}\right)=0 .
\end{aligned}
$$

Let us define $S(n)=\sigma_{\mathrm{s}}{ }^{2}(n) / \sigma_{\omega}{ }^{2}(n)$ as a signal to noise ratio $(S N R)$. Solving the quadratic equation (14), two normalized solutions are obtained as

$$
\frac{\gamma_{1 / 2}(n)}{\sigma_{\mathrm{c}}^{2}(n)}=\frac{1 \pm \sqrt{1+\frac{4}{N} \frac{(2+S(n))}{S(n)} \ln \left(\frac{\alpha}{1-\alpha}(1+S(n))\right)}}{2+S(n)} .
$$

Determined decision threshold must be a real number, non-negative, and larger than the estimated noise variance. Accordingly, discriminant of (15) must be non-negative. If the discriminant in (15) is in the range $[0,1]$, two solutions for the decision threshold are obtained. Decision threshold must be positive, so in the case that the discriminant in (15) is larger than 1, only one solution for the decision threshold is possible. If the sensing time in the process of spectrum sensing is predefined, the design goal for the CR, which is expressed through the weighting factor $\alpha$, must be adjusted to the environmental conditions.

Having in mind satisfaction of the requested conditions for the decision threshold and analyzing (15), it is determined that weighting factor $\alpha$ must satisfy

$$
\alpha>\frac{1}{2+S(n)}
$$

at any time instant $n$. In this case, determined decision threshold $\gamma(n)$ as a solution of (15) is unique and is given by 
$\gamma(n)=\sigma_{\mathrm{c}}^{2}(n) \frac{1+\sqrt{1+\frac{4}{N} \frac{(2+S(n))}{S(n)} \ln \left(\frac{\alpha}{1-\alpha}(1+S(n))\right)}}{2+S(n)}$.

In all the other cases, the design goal (weighting factor $\alpha$ ) should be modified and adjusted to its dynamic environment according to (16).

Finally, the proposed algorithm for the threshold determination and adaptation of the energy detector based spectrum sensing is described below:

Algorithm: Threshold adaptation algorithm for the energy detector-based spectrum sensing

1: Calculate the average energy of the received CR signal using (2).

2: Calculate the unbiased estimate of the autocorrelation coefficients $R_{\mathrm{S}}(j)$.

3: Compute the AR parameters using the least squares procedure and verify whether the Yule-Walker equations (11) and (12) are satisfied.

4: Find the variance of the detected signal $\sigma_{\mathrm{c}}{ }^{2}(n)$, and estimate the noise power $\sigma_{\omega}^{2}(n)$ from (13).

5: Estimate the PU signal power $\sigma_{\mathrm{s}}^{2}(n)$ from (9).

6: Verify that assumed weighting factor satisfies (16).

7: If yes, the optimal threshold value $\gamma(n)$ is determined according to (17). If no, the design goal must be modified and adjusted; get back to step 6 .

For application of the proposed algorithm in practice, it is important to estimate the computational burden by determining the algorithm order of complexity. The number of samples used by CR, $N$, directly influences the spectrum sensing time, which is directly connected to the spectrum usage efficiency of CRs, as well. Analyzing the algorithm, the order of complexity of with respect of the number of samples $N$ is obtained as $\mathrm{O}(N)$, which indicates efficient implementation. It is important to note that the closed form solution always leads to the threshold value in a finite number of steps, in contrast to iterative procedures.

\section{Simulation Results}

Evaluation of the proposed dynamic spectrum sensing algorithm is performed by 1200 Monte-Carlo simulations. Array of 1000 16-QAM symbols [23], which are considered as the PU signals, were generated and passed through the channel with AWGN. The AWGN has unknown SNR features, and its variance should be estimated by the CR assuming AR model with the order parameters $p=2$ and $q=78$ [18]. Weighting factor $\alpha=0.2$.

Figure 2 illustrates the dynamic adaptation of the decision threshold for various levels of $S N R$. From Fig. 2, the decision threshold is dynamically changing according to (17), depending on the $S N R$ in the environment. The first 400 simulated PU signals (array of 16-QAM symbols) were exposed to AWGN, with $S N R=0 \mathrm{~dB}$. For the next 400 simulations, $S N R$ was increased for $3 \mathrm{~dB}$. Finally, for the last 400 simulations, $S N R$ was decreased for $6 \mathrm{~dB}$. It should be noted that the value of the decision threshold is lower for higher $S N R$ and vice versa. For each simulation, weighting factor $\alpha$ satisfied (16).

\section{Conclusion}

In this paper, an algorithm for the decision threshold determination and adaptation in energy-based spectrum sensing is proposed. The algorithm is based on the noise and the PU signal power estimation through the usage of AR model and Yule-Walker high order equations. The closed-form solution for the optimal decision threshold is proposed and analyzed. Simulation results confirmed effectiveness of the proposed method for optimizing the sensing threshold as well as the capability for dynamic adaptation according to the changes in the operating environment. Also, order of complexity of the algorithm indicates efficient implementation.

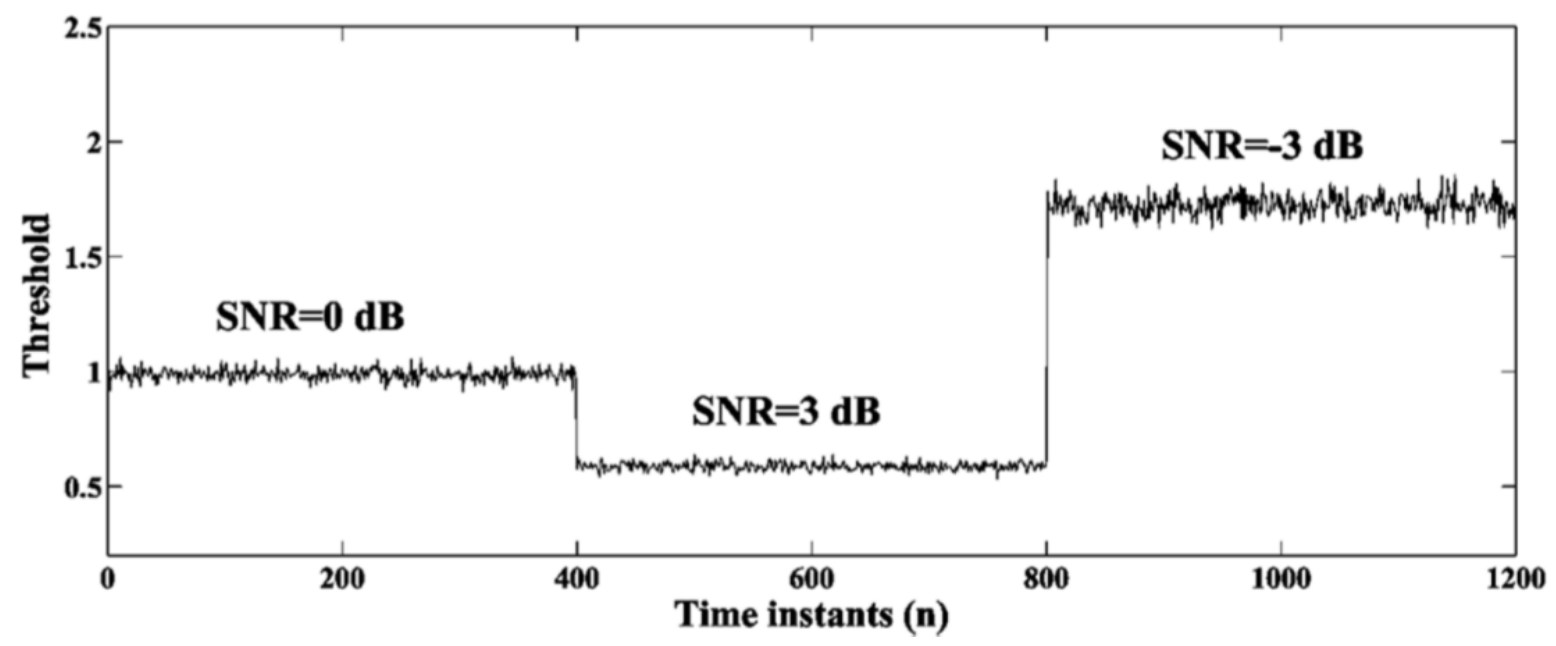

Fig. 2. Dynamic threshold in various SNR environments, for 16-QAM PU signals, AWGN propagation channel. 


\section{Acknowledgments}

This work was supported by the Serbian Ministry of Science under technology development project TR32028 "Advanced Techniques for Efficient Use of Spectrum in Wireless Systems".

\section{References}

[1] HAYKIN, S. Cognitive radio brain-empowered wireless communications. IEEE Journal on Selected Areas in Communications, 2005, vol. 23, no. 2, p. 201-220. DOI: 10.1109/JSAC.2004.839380

[2] MITOLA, J., MAQUIRE, Jr. G. Q. Cognitive Radio: making software radios more personal. IEEE Personal Communications, 1999, vol. 6, no. 4, p. 13-18. DOI: $10.1109 / 98.788210$

[3] AKYILDIZ, I. F., LEE, W. Y., VURAN, M., et al. Next generation/ dynamic spectrum access/ cognitive radio wireless networks: A survey. Computer Networks Journal, 2006, vol. 50, no. 13 , p. 2127-2159. DOI: 10.1016/j.comnet.2006.05.001

[4] CABRIC, D., MISHRA, S. M., BRODERSEN, R. W. Implementation issues in spectrum sensing for cognitive radios. In Proceedings of the 38th Asilomar Conference on Signals, Systems and Computers. Pacific Groove (USA), 2004, vol. 1, p. 772-776. DOI: 10.1109/ACSSC.2004.1399240

[5] YUCEK, T., ARSLAN, H. A survey of spectrum sensing algorithms for cognitive radio applications. IEEE Communications Surveys \& Tutorials, 2009, vol. 11, no. 1, p. 116-130. DOI: 10.1109/SURV.2009.090109

[6] MA, J., LI, G. Y., JUANG, B. H. Signal processing in cognitive radio. Proceedings of the IEEE, 2009, vol. 97, no. 5, p. 805-823. DOI: 10.1109/JPROC.2009.2015707

[7] SUTTON, P. D., NOLAN, K. E., DOYLE, L. E. Cyclostationary signatures in practical cognitive radio applications. IEEE Journal on Selected Areas in Communications, 2008, vol. 26, no. 1, p. 13 to 24. DOI: $10.1109 / \mathrm{JSAC} .2008 .080103$

[8] CABRIC, D., TKACENKO, A., BRODERSEN, R. W Experimental study of spectrum sensing based on energy detection and network cooperation. In Proceedings of the $1^{\text {st }}$ International Workshop on Technology and Policy for Accessing Spectrum (TAPAS). Boston (MA, USA), $2006 . \quad$ DOI: $10.1145 / 1234388.1234400$

[9] VARTIAINEN, J., LEHTOMAKI, J. J., SAARNISAARIS, H Double-threshold based narrowband signal extraction. In Proceedings of Vehicular Technology Conference. 2005, vol. 2, p. 1288-1292. DOI: 10.1109/VETECS.2005.1543516

[10] HAN, W., LI, J., Li, Z., et al. Double thresholds for judgment in cognitive spectrum sensing. In Proceedings of IEEE 19th International Symposium on Personal, Indoor and Mobile Radio Communications (PIMRC 2008). Cannes (France), 2008, 5 p. DOI: 10.1109/PIMRC.2008.4699894

[11] MISHRA, S. M., SAHAI, A., BRODERSEN, R. W. Cooperative sensing among Cognitive Radios. In Proceedings of International Conference on Communications ICC. Istanbul (Turkey), 2006, vol. 4, p. 1658-1663. DOI: 10.1109/ICC.2006.254957

[12] WANG, N., GAO, Y., ZHANG, X. Adaptive Spectrum Sensing Algorithm Under Different Primary users Utilizations. IEEE Communications Letters, 2013, vol. 17, no. 9, p. 1838-41. DOI: 10.1109/LCOMM.2013.081313.131468

[13] ABBassi-MOghadAM, D., MOHEBBI, A., MOHADES, Z. Performance analysis of time reversal UWB communication with non-coherent energy detector. Wireless Personal Communications,
2014, vol. 77, no. 3, p. 2291-2303. DOI: $10.1007 /$ s11277-0141638-x

[14] SEMLALI, H., BOUMAZZ, N., SOULMANI, A., et al. Energy detection approach for spectrum sensing in cognitive radio systems with the use of random sampling. Wireless Personal Communications, 2014, vol. 79, no. 2, p. 1053-1061. DOI: $10.1007 /$ s 11277-014-1917-6

[15] NAMDAR, M., ILHAN, H., DURAK-ATA, L. Optimal detection thresholds in spectrum sensing with receiver diversity. Wireless Personal Communications, 2016, vol. 87, no. 1, p. 63-81. DOI: $10.1007 / \mathrm{s} 11277-015-3026-6$

[16] CHATTERJEE, S., MAITY, S. On optimal threshold selection in cooperative spectrum sensing for cognitive radio networks: an energy detection approach using fuzzy entropy maximization. Wireless Personal Communications, 2015, vol. 84, no. 3, p. 1605 to 1625. DOI: $10.1007 / \mathrm{s} 11277-015-2550-8$

[17] KOZAL, A. S. B., MERABTI, M., BOUHAFS, F. An improved energy detection scheme for cognitive radio networks in low SNR region. In Proceedings of IEEE Symposium on Computers and Communications ISCC. Cappadocia (Turkey), 2012, p. 684-689. DOI: 10.1109/ISCC.2012.6249377

[18] JOSHI, D. R., POPESCU, D. C., DOBRE, O. A. Gradient-based threshold adaptation for energy detector in cognitive radio systems. IEEE Communications Letters, 2011, vol. 15, no. 1, p. 19-21. DOI: 10.1109/LCOMM.2010.11.100654

[19] CABRIC, D. Cognitive Radio: System Design Perspective (Ph. D. dissertation). University of California, Berkley, USA, 168 pages. [Online], Cited 2007-12-17. Available at: http://digitalassets.lib.berkeley.edu/techreports/ucb/text/EECS2007-156.pdf

[20] ZHANG, S., BAO, Z. An adaptive spectrum sensing algorithm under noise uncertainty. In Proceedings of International Conference on Communications ICC. Kyoto (Japan), 2011, 5 p. DOI: $10.1109 /$ icc.2011.5962493

[21] PALIWAL, K. K. Estimation of noise variance from the noisy AR signal and its application in speech enhancement. In Proceedings of IEEE International Conference on Acoustics, Speech, and Signal Processing (ICASSP '87). Dallas (USA), 1987, p. 297-300. DOI: 10.1109/ICASSP.1987.1169682

[22] CADZOW, J. A. Spectral estimation: An overdetermined rational model equation approach. In Proceedings of the IEEE, 1982, vol. 70, no. 9, p. 907-939. DOI: 10.1109/PROC.1982.12424

[23] HARADA, H., PRASAD, R. Simulation and Software Radio for Mobile Communications. Norwood (MA, USA): Artech House, 2002, ISBN: 1580530443

\section{About the Authors ...}

Rade BOZOVIC was born in Berane, Montenegro, in 1983. He received M.S. degree in Electrical Engineering from the Faculty of Electrical Engineering, University of Belgrade, in 2007. He is Ph.D. candidate at the Telecommunication Department at the Faculty of Electrical engineering, University of Belgrade since 2011. His research focuses in wireless communication systems, cognitive radio, signal processing. He is a radio engineer at CRONY, specialized for wireless communications.

Mirjana SIMIC was born in Sabac, Serbia, in 1973. She received the B.S., M.S. and the Ph.D. degrees in Electrical Engineering from the University of Belgrade, in 1998, 2006, and 2010, respectively. She is an Associate Professor 
at the Dept. of Telecommunications and Information Technology, University of Belgrade. Her research interests are in radio communications, cognitive radio, positioning in wireless communication systems, and location based services.

Predrag PEJOVIC was born in Belgrade, Serbia, in 1966. He received the B.S. and M.S. degrees in Electrical Engineering from the University of Belgrade, in 1990 and 1992, respectively, and the Ph.D. degree from the University of Colorado, Boulder, in 1995. In 1995, he rejoined the University of Belgrade, where he is presently a Professor at the Dept. of Electronics. His research interests are in analog circuit design, three-phase high power factor rectifiers, dynamics of nonlinear systems, electronic measurements, techniques for computer-aided analysis and design of power electronic systems, positioning in wireless communication systems, and location based services.
Miroslav L. DUKIC received the B.Sc. degree in Electronics and Communications in 1973 and the Ph.D. degree in 1981, all from the Faculty of Electrical Engineering, University of Belgrade. In 1973 he joined the Faculty of Electrical Engineering. Since 1973, he has taught several courses in communications theory, radar systems design, and modern electronic warfare systems. Professor Dukic was the head or the leading designer in over 150 big projects and studies for different customers, and has published a large number of papers in the field of communications. Professor Dukic's research interests include communications theory and signal processing for wireless communications systems, especially in the area of spread spectrum technology, radio communications and cognitive radio. He is a coauthor of several monographs, textbook Principles of Communications and he is the coauthor of the book Wireless Local Loops: Theory and Applications, published by Wiley. 